\title{
Spiking of the tibial tubercles-a radiological feature of osteoarthritis?
}

\author{
Simon Donnelly, Deborah J Hart, David V Doyle, Tim D Spector
}

\begin{abstract}
Objective-To determine whether 'spiking' or angulation of the tibial tubercle is associated with other radiographic markers of osteoarthritis (OA) or pain in the knee joint, and could be taken as a reliable marker for early $\mathrm{OA}$, in a large general population sample.

Methods-A total of 950 women from the Chingford general population survey underwent anteroposterior extended weight bearing radiography of the knees. Angulation of the tip of the medial and lateral tubercles, and height of the tubercles above the tibial plateau were measured. These measures were compared with standard radiographic indices including qualitative Kellgren and Lawrence global score, individual scores of osteophytes and joint space narrowing, and pain score. Intraobserver and interobserver reproducibility for assessment of spiking was tested in a subgroup of 50 films using two observers and two readings. Tibial spiking (angulation and height) was defined for this study as the top 10th centile for the whole population. Patients with normal radiographs (Kellgren and Lawrence grade 0 ) were allocated to quartile groups on the basis of spiking to define severity. Odds ratios were then calculated for the association of spiking and knee pain.
\end{abstract}

Results-The majority of the measures of tibial spiking were highly reproducible. There was a significant correlation between tibial spike angulation and the presence of osteophytes, but not joint space narrowing. The correlations for spike height with osteophytes and joint space narrowing were poor. There was an association between spike angulation at the lateral tubercle and reported knee pain (odds ratio 1.45 (95\% confidence interval 1.03 to 2.03)) after adjustment for age, body mass index, and Kellgren and Lawrence score. There was no association between medial spike angulation or spike height and pain. Among the 950 women, $683(72 \%)$ had normal radiographs (Kellgren and Lawrence $=0$ ); in this group there was a similar association between pain and lateral spike angulation, but not medial spike angulation or spike height.

Conclusions-Tibial spiking is associated with the presence of knee osteophytes and is reproducible, but does not have a strong independent relationship with knee pain. In patients with normal radiographs there is no useful correlation between tibial spiking and pain. Isolated tibial spiking is not a reliable sign of early knee $O A$, and should not routinely be reported.

(Ann Rheum Dis 1996; 55: 105-108)

The diagnosis of osteoarthritis (OA) of the knee for epidemiological purposes is traditionally based upon the radiographic appearance. Population surveys have shown that radiographically determined $\mathrm{OA}$ of the knee is present in between 14 and $30 \%$ of subjects older than 45 years and thereafter increases steadily with age. ${ }^{12}$ The most widely used definition, the Kellgren and Lawrence scale, ${ }^{3}$ places particular emphasis on the presence of osteophytes rather than joint space narrowing; osteophytosis is the most prevalent radiographic feature of OA at the knee joint. ${ }^{4}$ Recent studies have demonstrated the presence or absence of osteophytes to be the most reliable radiological marker for OA of the knee in the general population. ${ }^{56}$

The most important aspect of any early definition of $\mathrm{OA}$ is reliability in the detection of early disease features; early definition in OA is important for all studies of secondary disease prevention. It has been reported in radiological texts that 'spiking' or angulation of the tibial tubercles is a reliable marker of early OA of the knee, ${ }^{78}$ and one study 9 has demonstrated peaking of the tibial tubercles as a feature of established OA in a study of 55 patients. The aim of the present study was to ascertain whether isolated spiking of the tibial tubercle, as frequently reported by radiologists, is a reliable or useful marker for the presence of early OA in the knee.

\section{Patients and methods}

A total of 1003 women aged 45-65 years, selected from the age and gender register of a large general practice in Chingford, East London, were identified as described previously. ${ }^{10}$ The overall response rate for the group at initial recruitment was $78 \%$. This population is representative of the general female population of the United Kingdom in respect of height, weight, cigarette consumption, and alcohol intake. All the women lived within a five mile radius of the general practice; $98 \%$ were white; $42 \%$ were middle class white collar worker status (group C1), 32\% group $\mathrm{A} / \mathrm{B}, 17 \%$ group $\mathrm{C} 2$ and $8 \%$ group $\mathrm{D} / \mathrm{E}$ (Acorn classification, CACI International, London). All women completed a standardised nurse 


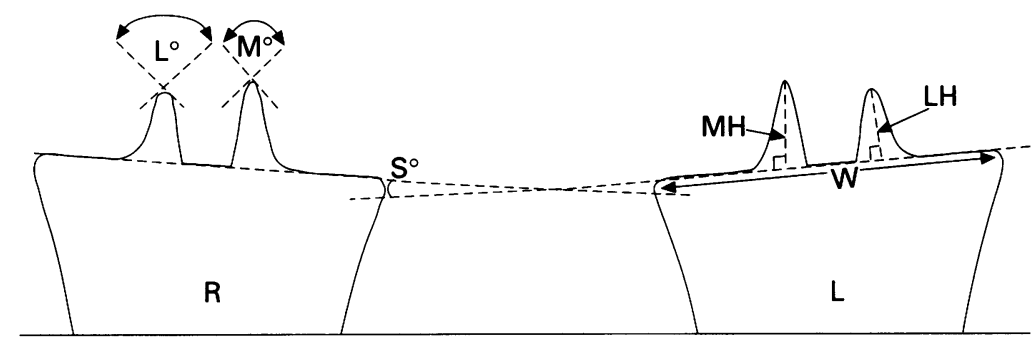

Measurement of tibial spike angulation and height. Tangents are drawn as close as possible to the tips of the medial and lateral tubercles and the angles (in degrees) are measured at the points of intersection. $L^{\circ}=$ Lateral spike angle; $M^{\circ}=$ medial spike angle. The width of the tibial plateau (W) and the heights of the lateral tubercle (LH) and medial tubercle (MH) above the plateau were measured. The ratio of the height of each tubercle above the tibial plateau to the overall width of the tibial plateau was calculated (lateral height $=L H / W$, medial height $=M H / W)$. Knee deformity was assessed as the angulation (in degrees) between an intersect drawn in parallel to each tibial plateau $\left(S^{\circ}\right)$

administered questionnaire. This included details of current and previous (aged 30 years) physical activity, in addition to physical demands of occupation or employment. Height was recorded in metres and weight in kilograms, to produce a body mass index (BMI) in $\mathrm{kg} / \mathrm{m}$.

Standardised weight bearing anteroposterior radiographs taken with the knee in the fully extended position were available from 950 women, allowing individual analysis of 1899 knees (one women from the study population had previously had one knee replacement). A single observer blinded to clinical details measured tibial spiking for each radiograph as described previously. ${ }^{9}$ Each radiograph was transposed to a transparent tracing sheet to produce measures of four tibial spikes and two tibial plateaus: lines were drawn on a tracing sheet fixed over each radiograph at a tangent to the tip of each tubercle on either side, intersecting at the tip of the tubercle, and the angle between these lines was measured (figure); the ratio of the height of each tubercle above the tibial plateau to the overall width of the plateau was calculated (figure). This measurement allowed correction for individual variation in tibial size. Angulation and height of the tibial tubercles were measured as hypersharpening of the tip of the spine in a vertical direction, rather than medial or lateral displacement of the tip of the spine. Finally, an intercept was drawn overlying both tibial plateaus and the angle between each intercept was taken to represent a measure of valgus or varus deformity at the knee. Intercepts that met at an angle below the midline of the tibial spikes were arbitrarily assigned a positive value (in degrees) (valgus), and those above, a negative value (varus).

A separate trained observer, blinded to clinical details and the above measures, scored each radiograph for individual features by reference to a standard altas at a single session as follows: osteophytes on a scale of $0-3$ for medial and lateral compartments; ${ }^{11}$ joint space narrowing on a scale of 0-3 for both compartments; ${ }^{11}$ global grading scale of Kellgren and Lawrence (0-4 scale $\left.{ }^{3}\right)$. Tibial spiking was then compared with standard radiographic measures using analysis of covariance (SAS software).
The intraobserver and interobserver reproducibility for measures of tibial spiking was tested on a subgroup of 50 knees randomly selected from the cohort. After a combined training session these were read as detailed above on separate occasions at an interval of two weeks. All repeat readings were made by observers blind to the original assessment, and no markings were allowed to be made on the radiographs on any occasion.

To define spiking, cutoffs for grades of spike height and angulation were chosen to approximate the 10th centile of their distribution in the whole population. The subpopulation with normal radiographs (Kellgren and Lawrence grade 0 ) were divided into quartiles based on spiking. The odds ratios for an association between spiking and knee pain could therefore be calculated. As an example, if the odds ratio was calculated to be $2 \cdot 0$ for this association, the presence of spiking in a subject would double the likelihood of that patient reporting knee pain.

As previous studies have demonstrated obesity to be a significant risk factor for knee $\mathrm{OA},{ }^{12}{ }^{13}$ the women were divided into tertiles of BMI for the whole population. The association between tibial spiking and the upper tertile of obesity was compared with that for the middle and lower tertiles.

\section{Results}

Table 1 shows the reproducibility of measures within and between the two observers. There was good agreement for most measures, with $\kappa$ values greater than 0.6 for most readings. Reproducibility was poorest for measurement of lateral spike angulation, with $\kappa$ values between 0.5 and 0.6 . Table 2 shows a comparison of tibial spike angulation and height with other radiological indices of OA. There was a strong association between tibial spike angulation and Kellgren and Lawrence score or peripheral osteophyte score. Lateral spike angulation showed a correlation with medial, but not lateral, joint space narrowing and medial spike angulation was not associated with joint narrowing. The correlation for spike height and other radiological features was weaker.

The association between obesity and knee pain (both important features of knee OA) and the presence of tibial spiking was examined (table 3 ). There was a significant reduction in spike angulation in association with obesity $(p<0.001$ for comparison of the upper and lower tertiles of obesity at the medial and lateral angles) and a corresponding increase in spike height that was weaker $(p<0.01$ at the medial and lateral heights). In patients who

Table 1 Reproducibility of radiographic scoring measures (50 films read twice) using weighted $\kappa$ statistics

\begin{tabular}{lll}
\hline Measures & $\begin{array}{l}\text { Intraobserver } \\
\text { agreement } \mathrm{K}\end{array}$ & $\begin{array}{l}\text { Interobserver } \\
\text { agreement } \mathrm{K}\end{array}$ \\
\hline Medial spike angle & 0.82 & 1.00 \\
Lateral spike angle & 0.55 & 0.51 \\
Medial spike height & 0.88 & 0.83 \\
Lateral spike height & 0.78 & 0.60 \\
\hline
\end{tabular}


Table 2 Comparison of tibial spike angulation and height with standard radiographic measures of knee osteoarthritis (adjusted for age and BMI)

\begin{tabular}{|c|c|c|c|c|c|c|c|c|c|}
\hline \multirow[t]{2}{*}{ Scoring system } & \multirow[t]{2}{*}{ Disease status } & \multicolumn{4}{|c|}{ Spike angles (deg) } & \multicolumn{4}{|c|}{ Spike height (mm) } \\
\hline & & Medial & $p$ & Lateral & $p$ & Medial & $p$ & Lateral & $p$ \\
\hline \multirow[t]{2}{*}{ Kellgren and Lawrence } & $0-1(n=1733)$ & $88 \cdot 2(20 \cdot 2)$ & \multirow{2}{*}{$<0.001$} & $100 \cdot 3(26 \cdot 3)$ & \multirow[b]{2}{*}{$<0.001$} & $0.138(0.03)$ & \multirow[b]{2}{*}{$0 \cdot 74$} & $0.125(0.04)$ & \multirow{2}{*}{0.003} \\
\hline & $2+(n=166)$ & $81 \cdot 5(26 \cdot 3)$ & & $85 \cdot 5(29 \cdot 7)$ & & $0.138(0.03)$ & & $0.135(0.03)$ & \\
\hline \multirow[t]{2}{*}{ Medial osteophyte } & $0(n=1776)$ & $88.0(20 \cdot 3)$ & \multirow{2}{*}{$<0.001$} & $99.9(26.6)$ & \multirow{2}{*}{$<0.001$} & $0.138(0.03)$ & \multirow{2}{*}{0.02} & $0.125(0.04)$ & \multirow{2}{*}{0.006} \\
\hline & $1+(n=123)$ & $80 \cdot 8(27 \cdot 3)$ & & $85.9(28.9)$ & & $0.148(0.03)$ & & $0.135(0.03)$ & \\
\hline \multirow[t]{2}{*}{ Lateral osteophyte } & $0(n=1757)$ & $88 \cdot 1(20 \cdot 6)$ & \multirow{2}{*}{$<0.001$} & $100 \cdot 2(26 \cdot 4)$ & \multirow{2}{*}{$<0.001$} & $0.138(0.03)$ & \multirow{2}{*}{0.36} & $0.125(0.04)$ & \multirow{2}{*}{0.002} \\
\hline & $1+(n=142)$ & $81 \cdot 3(24 \cdot 1)$ & & $83.8(28.2)$ & & $0.138(0.03)$ & & $0.138(0.03)$ & \\
\hline \multirow[t]{2}{*}{ Medial narrowing } & $0(n=1394)$ & $87.9(20.8)$ & \multirow{2}{*}{0.23} & $100 \cdot 1(26 \cdot 5)$ & \multirow{2}{*}{0.004} & $0.138(0.03)$ & \multirow{2}{*}{0.57} & $0.125(0.03)$ & \multirow{2}{*}{$0 \cdot 19$} \\
\hline & $1+(n=505)$ & $86 \cdot 6(21 \cdot 5)$ & & $95.9(28.0)$ & & $0.138(0.03)$ & & $0.125(0.04)$ & \\
\hline \multirow[t]{2}{*}{ Lateral narrowing } & $0(n=1721)$ & $87 \cdot 71(20 \cdot 8)$ & \multirow{2}{*}{0.41} & $99 \cdot 1(27 \cdot 1)$ & \multirow{2}{*}{0.57} & $0.138(0.03)$ & \multirow{2}{*}{0.02} & $0.125(0.04)$ & \multirow{2}{*}{0.06} \\
\hline & $1+(n=178)$ & $86 \cdot 34(22 \cdot 7)$ & & $97.9(26 \cdot 7)$ & & $0.148(0.03)$ & & $0.125(0.03)$ & \\
\hline
\end{tabular}

Values in parentheses are SD.

Table 3 Association of tibial spiking with obesity (expressed in tertiles of body mass index) and knee pain in 1899 knees

\begin{tabular}{|c|c|c|c|c|c|c|}
\hline Measures & Score & Number & Medial spike angle & Lateral spike angle & Medial spike height & Lateral spike height \\
\hline BMI & $\begin{array}{l}1 \cdot 00 \\
2 \cdot 00 \\
3 \cdot 00\end{array}$ & $\begin{array}{l}624 \\
622 \\
653\end{array}$ & $\begin{array}{l}90 \cdot 5(19 \cdot 9)^{\star \star} \\
87 \cdot 2(21 \cdot 5) \\
85 \cdot 2(21 \cdot 2)^{\star \star \star}\end{array}$ & $\begin{array}{c}102.9(25.9)^{\star \star} \\
99.0(25 \cdot 6)^{\star} \\
95 \cdot 2(28.8)^{\star \star \star}\end{array}$ & $\begin{array}{l}0.14(0.02) \\
0.14(0.05) \\
0.14(0.02)^{\star \star}\end{array}$ & $\begin{array}{l}0.12(0.02) \\
0.13(0.05) \\
0.13(0.03)^{\star \star}\end{array}$ \\
\hline Pain score & $\begin{array}{l}0 \\
1\end{array}$ & $\begin{array}{r}1412 \\
475\end{array}$ & $\begin{array}{l}87 \cdot 8(20 \cdot 3) \\
87 \cdot 7(22 \cdot 9)\end{array}$ & $\begin{array}{c}100 \cdot 0(26 \cdot 1) \\
95.6(29 \cdot 3)^{\star \star}\end{array}$ & $\begin{array}{l}0.14(0.03) \\
0.14(0.02)\end{array}$ & $\begin{array}{l}0.12(0.04) \\
0.13(0.03)\end{array}$ \\
\hline
\end{tabular}

Values are mean (SD) where relevant.

BMI = Body mass index.

Significance values for BMI $1 v$ BMI 2, BMI $2 v$ BMI 3, BMI $1 v$ BMI 3: ${ }^{\star} \mathrm{p}<0.05$; ${ }^{\star \star} \mathrm{p}<0.01$; ${ }^{\star \star} \mathrm{p}<0.001$

reported knee pain $(24 \cdot 8 \%)$ there was a reduction in spike angulation that reached significance at the lateral spine only, and no difference in spike height.

The odds ratios for pain in the top 10th centile for spiking were 1.45 (95\% confidence interval (CI) 1.03 to 2.03$)$ and $1.22(95 \% \mathrm{CI}$ 0.86 to 1.74$)$ at the lateral and medial angles, and $0.98(95 \%$ CI 0.69 to 1.39$)$ and 0.75 $(95 \% \mathrm{CI} 0.51$ to 1.09$)$ at the lateral and medial heights, respectively, after adjustments for age, BMI, and Kellgren and Lawrence score. The radiograph was normal in 683 patients $(71.9 \%)$ (Kellgren and Lawrence grade 0$)$. In this group the medial tubercle was more prominent than the lateral (height $0.14 \mathrm{~mm}$ $v 0.12 \mathrm{~mm}(\mathrm{p}<0.001)$, angle $89^{\circ} v 102^{\circ}$ $(p<0.001))$. These patients were assigned dichotomously to quartiles based on spike angulation and height (table 4). The odds ratios for pain in the highest quartile for spiking

Table 4 Odds ratio for pain in patients with normal radiographs (Kellgren and Lawrence score $=0)(n=1366$ knees $)$ after adjustment for age and BMI

\begin{tabular}{|c|c|c|c|}
\hline Feature & Quartiles & Number (\%) & $\begin{array}{l}\text { Odds ratio for pain } \\
(95 \% C I)\end{array}$ \\
\hline Medial spike height & $\begin{array}{l}1 \\
2 \\
3 \\
4\end{array}$ & $\begin{array}{l}310(22 \cdot 7) \\
349(25 \cdot 1) \\
336(24 \cdot 6) \\
377(27 \cdot 6)\end{array}$ & $\begin{array}{l}1.00 \\
1.26(0.85 \text { to } 1.83) \\
1.28(0.86 \text { to } 1.89) \\
1.04(0.69 \text { to } 1.54)\end{array}$ \\
\hline Lateral spike height & $\begin{array}{l}1 \\
2 \\
3 \\
4\end{array}$ & $\begin{array}{l}311(22 \cdot 8) \\
368(26 \cdot 9) \\
347(25 \cdot 4) \\
340(24 \cdot 9)\end{array}$ & $\begin{array}{l}1.00 \\
1.03(0.69 \text { to } 1.51) \\
1.10(0.74 \text { to } 1.63) \\
1.16(0.78 \text { to } 1.71)\end{array}$ \\
\hline Medial spike angle & $\begin{array}{l}1 \\
2 \\
3 \\
4\end{array}$ & $\begin{array}{l}322(23 \cdot 6) \\
328(24 \cdot 0) \\
337(24 \cdot 7) \\
379(27 \cdot 7)\end{array}$ & $\begin{array}{l}1.00 \\
1.07(0.74 \text { to } 1.55) \\
0.79(0.53 \text { to } 1.16) \\
1.11(0.77 \text { to } 1.61)\end{array}$ \\
\hline Lateral spike angle & $\begin{array}{l}1 \\
2 \\
3 \\
4\end{array}$ & $\begin{array}{l}303(22 \cdot 2) \\
351(25 \cdot 7) \\
334(24 \cdot 5) \\
378(27 \cdot 7)\end{array}$ & $\begin{array}{l}1.00 \\
1.06(0.72 \text { to } 1.54) \\
0.88(0.59 \text { to } 1.29) \\
1.47(1.02 \text { to } 2.13)\end{array}$ \\
\hline
\end{tabular}

were 1.47 and 1.11 at the lateral and medial angles, respectively, and 1.16 and 1.04 at the lateral and medial heights, respectively (table 4). There was no trend of valgus or varus deformity in association with other measures of OA (results not shown).

\section{Discussion}

This large population based survey has shown an association between spiking of the tibial tubercles and the presence of osteophytes, but there was no strong independent association with knee pain; an association was demonstrated for angulation, rather than height of the tibial spike. For epidemiological surveys, radiographic OA is best defined by the use of standard atlases, using an osteophyte score or the method of Kellgren and Lawrence, which also depends upon the presence of osteophytes. In our survey, both of these standardised methods showed an association with angulation of the tibial spike. This association was not found for joint space narrowing.

A previous study ${ }^{9}$ demonstrated spiking of the tibial tubercles as a feature of established knee OA in a sample of 55 patients. Although the numbers in this study were small, the reported association was strongest for angulation of the tibial spike, rather than height as in our patients. A review of the radiological features of $\mathrm{OA}$ in 1990 by Alexander ${ }^{14}$ suggested that peaking of the tibial tubercle as a feature of $O A$ is indistinguishable from an osteophyte arising at any other articular margin, and should therefore carry the same significance. Our findings in the present study give some support to the theory that tibial spiking is a form of osteophyte formation. 
Measurement of tibial spiking by the method described showed good interobserver and intraobserver reproducibility, with the exception of lateral spike angulation. We have previously demonstrated high reproducibility for standardised radiological measures of OA, including Kellgren and Lawrence score and the presence of osteophytes. ${ }^{5}$

The primary purpose of this study was to examine whether tibial spiking as an isolated radiological feature is indicative of $\mathrm{OA}$ at the knee. At present, we lack a gold standard of early radiological OA for comparison; however, most observers would agree that, for a radiological feature to be relevant to the disease, knee pain should be associated with that feature. We found an association between lateral spike angulation and the presence of pain, though this was not seen for medial spike angulation or spike height on either side. The odds ratio for pain using either a 10 th centile cutoff for spiking in the population as a whole, or the worst quartile for spiking in those with normal radiographs, confirmed a weak association between lateral spike angulation and the presence of pain. There was no association between medial spike angulation or spike height and pain. We have previously examined the association between knee pain and osteophyte formation ${ }^{5}$ and showed an odds ratio for pain of $3.73(95 \%$ CI 2.60 to 5.35$)$ in the presence of medial osteophytosis and 3.16 (95\% CI $2 \cdot 25$ to $4 \cdot 43$ ) for lateral osteophytosis (10th centile cutoff). Using a Kellgren and Lawrence score of 2+ to define knee OA in the same population, the odds ratio for pain was 3.61 (95\% CI $2 \cdot 63$ to $4 \cdot 95$ ). Although spiking of the lateral tibial tubercle is associated with osteophytosis, as an isolated feature it is only a weak predictor (odds ratio 1.47) for the presence of knee pain, and in our survey its reproducibility was poorest. The strongest radiological association with knee pain therefore remains the presence of osteophytes, either alone or as a feature of the Kellgren and Lawrence scale. In an assessment of the clinical significance of any radiographic marker as a feature of early OA, it can be argued that pain is not a consistent feature of early $\mathrm{OA}$ and therefore not a valid criterion for comparison. In this regard, arthroscopy or magnetic resonance imaging of the knee could provide earlier preclinical evidence of OA and serve for comparison. However, most epidemiological studies have identified pain as a consistent feature of knee OA that correlates with radiographic progression, ${ }^{515}$ and it has recently been demonstrated as an early feature of $\mathrm{OA}$ even in the absence of radiographic change. ${ }^{16}$

The association between tibial spiking and obesity in our study was not unexpected, given the association between obesity and both prevalence and progression of knee OA (as defined by osteophytes) in a number of studies. $^{1213}$ The association between tibial spiking and osteophytosis in the present study suggested that a similar association with obesity would result.

This cross sectional study has examined a large female population, and for completeness it would be necessary to evaluate tibial spiking further, longitudinally, in a population without OA (Kellgren and Lawrence $=0$ ), to include the occurrence of established OA (Kellgren and Lawrence $\geq 2$ ) in that population up to five years later. Similarly, arthroscopy or magnetic resonance imaging in patients with tibial spiking would detail actual articular cartilage loss as assessed indirectly by joint space narrowing in the present study.

In summary, our study has confirmed an association between spiking or peaking of the tibial tubercle and the presence of lateral and medial osteophytosis and obesity, with a weak independent association between knee pain and spike angulation at the lateral tubercle only. Isolated tibial spiking is therefore not a reliable marker for the presence of knee OA and should not routinely be reported.

1 Van Sasse J L C M, Van Romunde L K S, Cats A, Vandenbrouche J P, Valkenburg H A. Epidemiology of osteoarthritis: Zoetermeer study. Comparison of radiological osteoarthritis in a Dutch population with that
in 10 other populations. Ann Rheum Dis 1989; 48: in 10 .

2 Spector T D, Hart D J. How serious is knee osteoarthritis? Ann Rheum Dis 1992; 51: 1105-6.

3 Kellgren J K, Lawrence J S. Radiological assessment of osteoarthritis. Ann Rheum Dis 1957; 16: 491-501.

4 Thomas R H, Resnick D, Alazraki N P, Daniel D, Greenfield R. Compartmental evaluation of osteoarthritis of the knee. Radiology 1975; 116: 585-94.

5 Spector T D, Hart D J, Byrne J, Harris P A, Dacre J E, Doyle D V. Definition of osteoarthritis of the knee for epidemiological studies. Ann Rheum Dis 1993; 52: 790-4.

6 Altman R, Alarcon G, Appelrouth D. Criteria for classification and reporting of osteoarthritis of the knee. Arthritis Rheum 1995. In press.

7 Sutton D. A textbook of radiology and imaging, 4th edn. Edinburgh: Churchill Livingstone, 1987; 113.

8 Resnick D, Niwayama G. Diagnosis of bone and joint disorders, 2nd edn. Philadelphia: WB Saunders Co, 1988; 1448.

9 Reiff D B, Heron C W, Stoker D J. Spiking of the tubercles of the intercondylar eminence of the tibial plateau in osteoarthritis. Brf Radiol 1991; 64: 915-7.

10 Hart D J, Spector T D. The relationship of obesity, fat distribution and osteoarthritis in women in the genera population: the Chingford study. $\mathcal{F}$ Rheumatol 1993; 20 331-5.

11 Spector T D, Cooper C, Cushnaghan J, Hart J D, Dieppe P A. A radiographic atlas for knee osteoarthritis. London: Springer Verlag, 1992; 506-10.

12 Spector T D. The fat on the joint: osteoarthritis and obesity. 7 Rheumatol 1990; 17: 283-4.

13 Spector T D, Hart D J, Doyle D V. Incidence and progression of osteoarthritis in women with unilateral knee disease in the general population: the effect of obesity. Ann Rheum Dis 1994; 53: 565-8.

14 Alexander C J. Osteoarthritis: a review of old myths and current concepts. Skeletal Radiol 1990; 19: 327-33.

15 Ledingham J, Regan M, Jones A, Doherty M. Radiographic patterns and associations of osteoarthritis of the knee in patients referred to hospital. Ann Rheum Dis 1993; 52: $520-6$.

16 Brandt K D, Fife R S, Braunstein E M, Katz B. Radiographic grading of the severity of knee osteoarthritis: relation of the Kellgren and Lawrence grade to a grade based on joint space narrowing, and correlation with arthroscopic evidence of articular cartilage degeneration. Arthritis Rheum 1991; 34: 1381-6. 